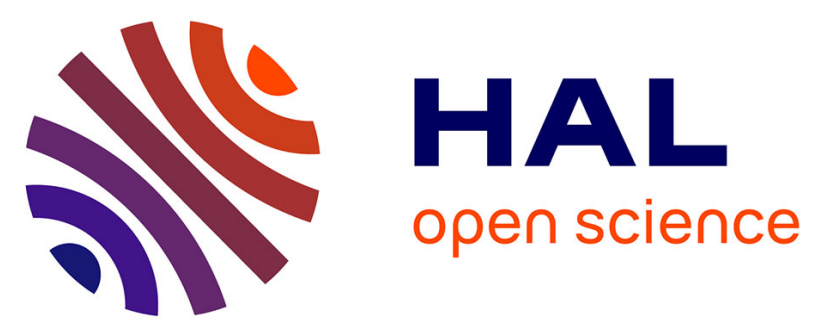

\title{
Light emission despite doubly-forbidden radiative transitions in AlP/GaP quantum wells: Role of localized states
}

S. Bhuyan, R. Mondal, P. Khatua, M. Semtsiv, W. T. Masselink, Jean Léotin, B. Pal, B. Bansal

\section{To cite this version:}

S. Bhuyan, R. Mondal, P. Khatua, M. Semtsiv, W. T. Masselink, et al.. Light emission despite doubly-forbidden radiative transitions in $\mathrm{AlP} / \mathrm{GaP}$ quantum wells: Role of localized states. Journal of Applied Physics, 2013, 114, pp.163101. 10.1063/1.4825328 . hal-00938395

\section{HAL Id: hal-00938395 \\ https://hal.science/hal-00938395}

Submitted on 21 Aug 2020

HAL is a multi-disciplinary open access archive for the deposit and dissemination of scientific research documents, whether they are published or not. The documents may come from teaching and research institutions in France or abroad, or from public or private research centers.
L'archive ouverte pluridisciplinaire HAL, est destinée au dépôt et à la diffusion de documents scientifiques de niveau recherche, publiés ou non, émanant des établissements d'enseignement et de recherche français ou étrangers, des laboratoires publics ou privés.

\section{(c)(1)}

Distributed under a Creative Commons Attribution| 4.0 International License 


\title{
Light emission despite doubly-forbidden radiative transitions in AIP/GaP quantum wells: Role of localized states
}

\author{
Sumi Bhuyan, ${ }^{1}$ Richarj Mondal, ${ }^{1}$ Pradip Khatua, ${ }^{1}$ Mykhaylo Semtsiv, ${ }^{2}$ W. T. Masselink, ${ }^{2}$ \\ Jean Léotin, ${ }^{3}$ Bipul Pal, ${ }^{1}$ and Bhavtosh Bansal ${ }^{1, a)}$ \\ ${ }^{1}$ Indian Institute of Science Education and Research Kolkata, Mohanpur, Nadia 741252, West Bengal, India \\ ${ }^{2}$ Department of Physics, Humboldt University Berlin, Newtonstrasse 15, D-12489 Berlin, Germany \\ ${ }^{3}$ Laboratoire National des Champs Magnétiques Pulsés, 143 avenue de Rangueil, 31400 Toulouse Cedex 4, \\ France
}

\begin{abstract}
The GaP/AlP/GaP heterostructure has an indirect gap both in real as well as momentum space, making the first order radiative recombination doubly forbidden. Nevertheless, we have observed relatively efficient emission from these structures. This paper comprehensively studies the origin of this improved light emission through a detailed analysis of the photoluminescence (PL) spectra. Our observations suggest that localized excitons within the acceptor states in GaP close to the heterostructure interface are enough for efficient light emission in these structures, doing away with the need for more complicated structures (superlattices or neighboring confinement structures). This real space localization of holes, close to the interface, apart from increasing the wave function overlap, also relaxes the delta-function momentum selection rule. Independent experimental evidence for this assertion comes from (i) the PL spectrum at high excitation power where transitions from both the localized as well as extended states are independently observed, (ii) the observation that extended states emission has the expected band-bending-induced blue-shift with increase in excitation power, whereas the localized states do not, (iii) observation of phonon replicas for PL from localized states, and (iv) observation of persistent photoconductivity at low temperature. Finally, we propose a simple analytical model that accounts for both the type-II nature as well as the indirect bandgap to explain the improvement of radiative recombination efficiency with increased localization. The experimental observations are reproduced within an order of magnitude. The model is very general and it also provides a framework to study the optical properties of other such (type-II and/or indirect gap) heterostructures.
\end{abstract}

\section{INTRODUCTION}

Despite the near lattice match, AlP/GaP is among the least studied of the group III-V heterojunction pairs. This is because both AlP and GaP have an indirect gap. To make things bleaker for optoelectronics, the band alignment between $\mathrm{AlP}$ and $\mathrm{GaP}$ is type-II; the $\mathrm{X}$-valley electrons ( $\Gamma$ valley holes) see a lower potential in AlP (GaP) [Fig. 1]. As a result, the overlap of electron and hole wave functions is not expected to be optimal. Non-optimal overlap would lead to poor radiative efficiency.

Due to the expected poor emission efficiency, AlP-based materials have been largely ignored. The little research reported, mostly in 1990's, has primarily focused on innovative bandgap engineering ideas which may improve the poor light emitting efficiency expected of these structures. Most studies have been made on $(\mathrm{AlP})_{m} /(\mathrm{GaP})_{n}$ superlattices, where $m$ and $n$ are the number of monolayers. ${ }^{1-5}$ Like for the $\mathrm{Si} / \mathrm{Ge}$ system, zone folding is predicted to yield a direct gap also in AlP/GaP for suitable $m$ and $n \cdot{ }^{1,6}$ Relatively, efficient light emission has indeed been observed from these structures but conclusive evidence for direct transitions has never been found. It is now generally believed that the

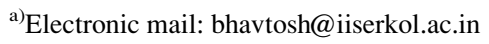

disorder-related relaxation of the momentum selection rules and carrier localization are responsible for unexpected bright emission from these samples. Other ideas for improving light emission include making the AlP quantum well (QW) only a few atomic layers thin so that there may be large spillover of the electron wave function outside the AIP QW and the $\Gamma$-X valley mixing due to the abrupt heterointerface may lead to a phonon-free pathway for light emission. ${ }^{7,8}$ Furthermore, for the GaP/AlP/GaP type-II QWs, since the holes in GaP would effectively be free, neighboring confinement systems to confine the holes have also been tried. ${ }^{9-11}$ Finally, there was also a report of insertion of an ultrathin AlP QW between the GaAsP/GaP heterostucture to improve the radiative efficiency of $\mathrm{GaP} .{ }^{12}$ In most of these studies, a relatively efficient photoluminescence (PL) has been observed which has been tentatively attributed to carrier localization. ${ }^{4,11}$ But this localization and its origin have never been clearly established. It is hence surprising that apart from some very preliminary work, ${ }^{13}$ there is no reported luminescence study on more conventional, wider QWs of AlP/GaP. Without the added complications of the above mentioned engineering ideas, wider QWs are perhaps better suited to probe the nature of localization. In this paper, we have studied GaP/AlP/GaP multiple QW (MQW) samples of 3, 4, and $5 \mathrm{~nm}$ well widths. Through a systematic study of the PL in 


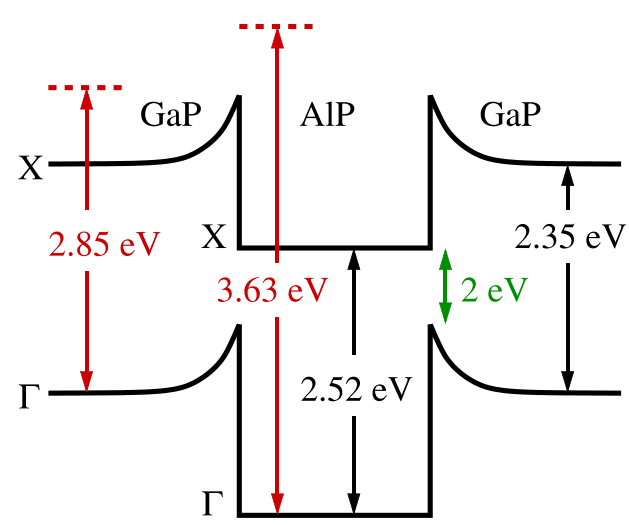

FIG. 1. Schematic band diagram of GaP/AlP/GaP QWs under low optical excitation. Note that the spatially-indirect optical transition between Xvalley electrons in AIP and holes in GaP occurs at energy smaller than the bandgaps of both GaP and AlP.

these MQWs, we have attempted to elucidate the primary mechanism responsible for the relatively efficient light emission.

\section{EXPERIMENT AND OBSERVATIONS}

Multiple quantum wells composed of Si-doped AlP wells and GaP barriers were grown using gas-source molecularbeam epitaxy on unintentionally doped n-type $\mathrm{GaP}(001)$ substrates with $n \approx 8 \times 10^{15} \mathrm{~cm}^{-3}$ at $300 \mathrm{~K} .{ }^{14}$ Low temperature (15 K) PL spectra were measured by nonresonantly exciting the samples with a $405 \mathrm{~nm}$ diode laser with the power incident on the sample varied between $20 \mu \mathrm{W}$ and $20 \mathrm{~mW}$. The spectra were dispersed in a $0.5 \mathrm{~m}$ monochromator and were recorded using a silicon charge coupled device with a spectral resolution of $0.5 \mathrm{meV}$.

A simplified schematic band diagram of the AlP QW sandwiched between GaP layers is shown in Fig. 1. The type-II band alignment makes the transition energy $(\sim 2.01-2.05 \mathrm{eV})$ smaller than the energy gaps of both the parent compounds. For simplicity, the strain-induced splitting of the three-fold degenerate X-valley of AlP is not shown. ${ }^{15,16}$ It is important to note that there will be an electrostatically produced band-bending. ${ }^{17}$ This will be especially important for the holes in the GaP layer. The valence band holes should observe a carrier density-dependent confinement potential which may be approximated by a triangular well. ${ }^{17}$ It was perhaps not realized that this potential makes the extra QWs created in the neighboring confinement structures $^{9-11}$ redundant. There will also be an accompanying band bending in the AlP QW layer, but since this would only add a small perturbation to the already present strong QW confinement potential felt by electrons, the band bending inside the QW is not shown.

Figure 2 shows PL spectra from three different AlP/GaP MQW samples (well widths 3, 4, and $5 \mathrm{~nm}$ ). All the spectra have been scaled to the height of the strongest peak and the abscissa shifted such that the strongest peak is centered at zero energy. This clearly shows that all the three samples have a second peak at approximately the same energy ( $\sim 45 \mathrm{meV}$ ) below the first peak. This energy difference is close to various longitudinal optical (LO) phonon energies in

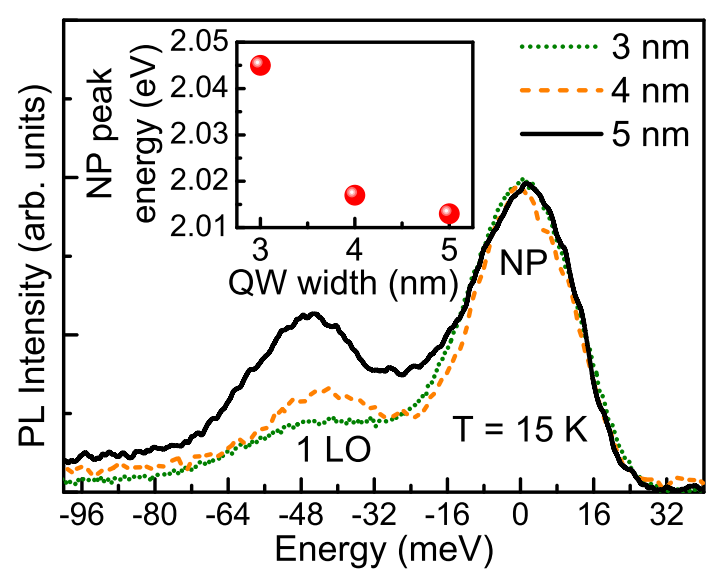

FIG. 2. Photoluminescence spectra from three AlP/GaP MWQ samples measured at $15 \mathrm{~K}$ under very low excitation power $(\sim 20 \mu \mathrm{W})$. The spectra have been scaled such that the prominent no-phonon (NP) peak in different samples has the same intensity and lies around zero energy. In each of the three cases, the lower intensity (phonon-assisted) peak is about $45-50 \mathrm{meV}$ below the NP peak. Note that the intensity of the phonon peak relative to the NP peak increases with increasing well width. (Inset) The actual energy position of the NP peak as a function of the well width. The blue-shift in the NP peak energy with decreasing well width ascertains that the electrons are indeed those confined in the AlP QWs.

$\mathrm{GaP}, 50 \mathrm{meV}$ at the $\Gamma$ point, and about $46 \mathrm{meV}$ at $\mathrm{L}$ and $\mathrm{X}$ valleys. ${ }^{18}$ Hence, the second lower energy peak is from a phonon-assisted transition and the first peak is identified as the no-phonon (NP) peak. Note that the wider QWs have relatively weaker NP peaks. The actual transition energies for NP peaks are shown in Fig. 2 (inset). The systematic blueshift of the spectra with the decreasing well width confirms quantum confinement of carriers and hence our first important conclusion is that at least the electrons participating in PL are indeed from the AlP QWs. This rules out the trivial defect recombination from $\mathrm{GaP}$ barrier layers alone.

Now onwards, we shall mostly be discussing the results from the $3 \mathrm{~nm}$ MQW sample. However, note that the other two samples (4 and $5 \mathrm{~nm}$ MQWs) also showed nearly identical results. Low temperature PL from the $3 \mathrm{~nm}$ MQW sample with the excitation power varied over three orders of magnitude is plotted in Fig. 3 on a semilogarithmic scale. Phonon replicas of the no-phonon peak due to longitudinal optical (LO) phononassisted indirect transitions are seen at all excitation powers. Tentatively, we assign these peaks as A-B(NP) [acceptor-toband-NP transition], A-B(LO) [acceptor-to-band-one LO phonon-assisted transition], and $\mathrm{A}-\mathrm{B}(2 \mathrm{LO})$ [acceptor-toband-two LO phonon-assisted transition], respectively. We shall justify the assignment of acceptor-to-band transitions in Sec. III. At higher powers, a second peak emerges at slightly higher energy than the $\mathrm{A}-\mathrm{B}(\mathrm{NP})$ peak. While the energy positions of the first three peaks are independent of the excitation power, the new peak, labeled as B-B(NP) [band-toband - NP transition] shows a blue-shift with power.

This is further analyzed in Fig. 4, where the powerdependent PL data are re-plotted in the inset on a linear scale. Here, the signal is normalized at the A-B(NP) peak for a clear comparison. It is evident that the energy position of the A-B peak is virtually constant with excitation power, whereas the B-B peak shows a clear blue-shift. The energy 


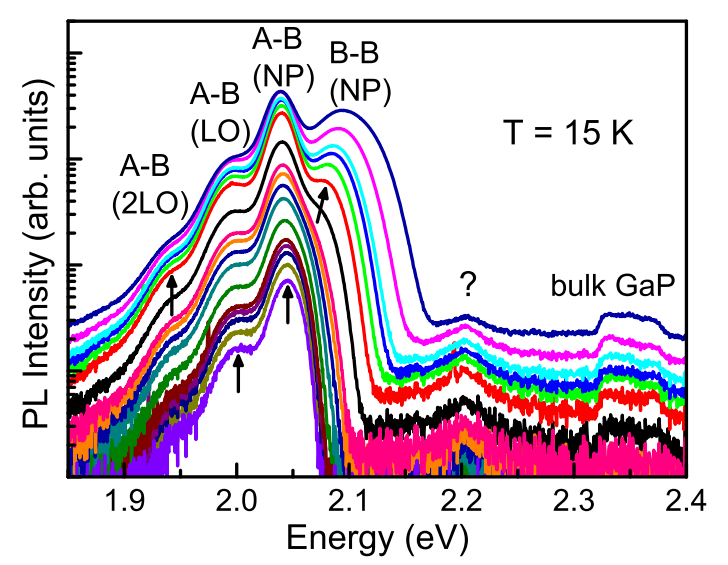

FIG. 3. Photoluminescence spectra from $3 \mathrm{~nm}$ MQW sample measured at $15 \mathrm{~K}$ as a function of excitation power $(20 \mu \mathrm{W}-20 \mathrm{~mW})$. The peaks due to NP acceptor-to-band [A-B(NP)], one LO phonon-assisted acceptor-to-band [A-B(LO)], two LO phonon-assisted acceptor-to-band [A-B(2LO)], and NP band-band $[\mathrm{B}-\mathrm{B}(\mathrm{NP})]$ transitions are marked. The A-B transitions do not show blue-shift, whereas the B-B(NP) transition is strongly blue-shifted with increasing excitation power. The peak at $\sim 2.2 \mathrm{eV}$ is not identified.

of B-B peak plotted in Fig. 4 as a function of [power] $]^{1 / 3}$ nicely fits a straight line. This is well-known in type-II systems ${ }^{19}$ and arises due to carrier induced band-bending and resultant nearly triangular potential well (schematically shown for the valance band in Fig. 1) whose steepness increases with the excitation power leading to the [power] $]^{1 / 3}$ dependence of the blue-shift of PL peak. ${ }^{17}$

The temperature dependence of the PL from the same $3 \mathrm{~nm}$ MQW sample measured under $5 \mathrm{~mW}$ excitation power is presented on a semi-logarithmic scale in Fig. 5. We observe a rapid drop in intensity of the three lower energy A-B peaks with increasing temperature and beyond $80 \mathrm{~K}$, only the high energy B-B peak can be clearly identified.

We also investigated time-resolved photoconductivity. The device was made with gold wires bonded on the sample surface to indium contacts which were annealed to make direct contact to the underlying QW layer. We measured in-plane two-probe photoresistance when the sample maintained at $15 \mathrm{~K}$ was exposed to $532 \mathrm{~nm}$ laser light. The time evolution of photoresistance was recorded as the light source

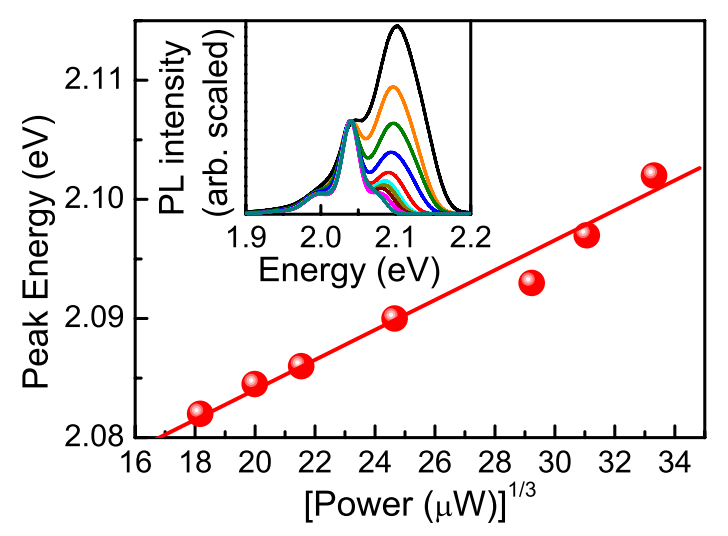

FIG. 4. Energy of the B-B(NP) peak in the $15 \mathrm{~K}$ PL spectra from $3 \mathrm{~nm}$ MQW sample is plotted as a function of [power] ${ }^{1 / 3}$. The solid line is a linear fit showing the expected [power] ${ }^{1 / 3}$ dependence of blue-shift. Inset: Power dependent PL spectra normalized at the A-B(NP) peak at $2.05 \mathrm{eV}$. Note that there is no energy shift in the A-B(NP) peak with power.

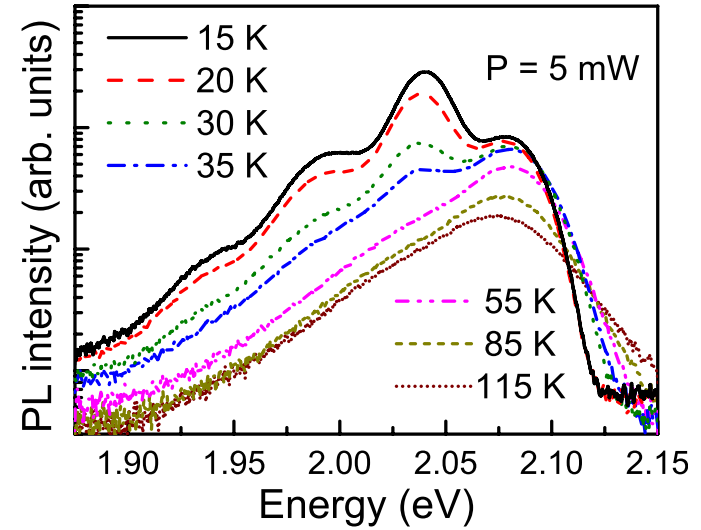

FIG. 5. Temperature dependence of the PL spectra for $3 \mathrm{~nm}$ MQW sample measured under $5 \mathrm{~mW}$ excitation power. Note that at higher temperature, band-to-band recombination dominates over the acceptor-to-band transitions.

was switched on and then abruptly switched off after a steady state in the light-on condition was reached. Timeresolved photoconductivity was inferred from the photoresistance measurement. Figure 6 shows the time evolution of normalized photoconductivity from $3 \mathrm{~nm}$ MQW sample. The decay of the photoconductivity $\sigma(t)$ after the abrupt switching off of light is replotted in Fig. 6 (inset). The experimental data can be fitted ${ }^{20}$ to the Kohlrausch stretched exponential form: $\sigma(t)=\sigma(0) \exp (-t / \tau)^{\beta}$ with $\beta=0.12$ and with a very large effective decay time $\tau \sim 3.5 \times 10^{7} \mathrm{~s}$ at $15 \mathrm{~K}$. Hence, we have observed persistent photoconductivity. ${ }^{20}$

\section{DISCUSSION}

\section{A. Evidences for hole localization}

We shall now try to infer the nature of the light emitting states from these observations. The first conclusion which had already been made earlier on the basis of the shift in the transition energy with well width [Fig. 2 (inset)] is that the electron states are quantized within the QW.

Let us now pay attention to the hole states. Based on experimental observations stated above, we shall argue in the following that at low excitation densities, the PL emission

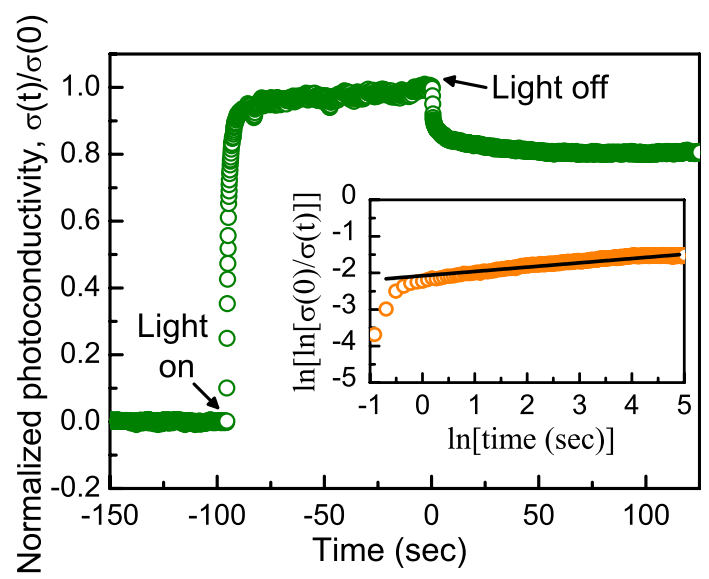

FIG. 6. Time evolution of normalized photoconductivity under abrupt switching on and off of a green $(532 \mathrm{~nm})$ laser light exciting the MQW sample at $15 \mathrm{~K}$. Inset: Decay of photoconductivity is fitted to Kohlrausch stretched exponential: $\sigma(t)=\sigma(0) \exp (-t / \tau)^{\beta}$ with $\beta=0.12$ and $\tau \sim 3.5 \times 10^{7} \mathrm{~s}$. 
involves the localized hole states in GaP leading to the localized hole-to-conduction band transition [A-B(NP)] in Fig. 3. Strong localization in real space leads to fuzziness in momentum owing to the position-momentum uncertainty principle. This facilitates electron-hole wave-function overlap in momentum space and enhances optical transition probability in indirect gap systems. This is schematically shown in Fig. 7. To ensure nonzero wave-function overlap in real space in the type-II systems, only the hole states localized very close to the QW interfaces can participate in PL. Such localization centers are much smaller in number than the band states. At high excitation powers, these get saturated and the band-to-band (B-B) emission also becomes visible. One may obtain a rough estimate of binding energy of these localized states from the difference in the observed PL energy of the B-B(NP) and A-B(NP) peaks. This is about $35 \mathrm{meV}^{21}$ and is most likely related to a shallow acceptor state. It is emphasized that unlike the type-I structures, here the holes cannot be localized by the interface fluctuations (there is no confinement potential for holes). The extent of the bound hole wave function, $d$, can be estimated from the uncertainty argument, $d \approx \hbar / \sqrt{2 m_{h} E_{B}} \cdot 22$ With the heavy hole mass in GaP $m_{h}=0.67 m_{0}$, where $m_{0}$ is the free electron mass, and localization energy $E_{B} \approx 35 \mathrm{meV}$, we get $d \approx 1.2 \mathrm{~nm}$, that is about two lattice constant. Such strongly localized hole states would have a considerable spread in their momentum states which would support phonon-free recombination in this indirect gap system. Even the electron states in these narrow QW samples are localized within the thin QW region. Stronger localization in narrower QWs leads to enhanced no-phonon transition relative to the phonon-assisted transitions compared with wider QWs as is seen in Fig. 2.

We have observed a large oscillator strength for the phonon satellites connected with the A-B transitions, whereas there are no phonon satellites at all for the B-B peaks. While in an indirect gap material, phonon-assisted transitions are expected, these are usually extremely weak because the long radiative recombination time associated with the second order pathway has to compete with nonradiative processes. On the other hand, localization of excitons has an effect of considerably enhancing oscillator strength of these satellites due to the increase in the Huang-Rhys factor. ${ }^{23}$ Notably, the absence of phonon satellites in the B-B(NP) peak leads us to

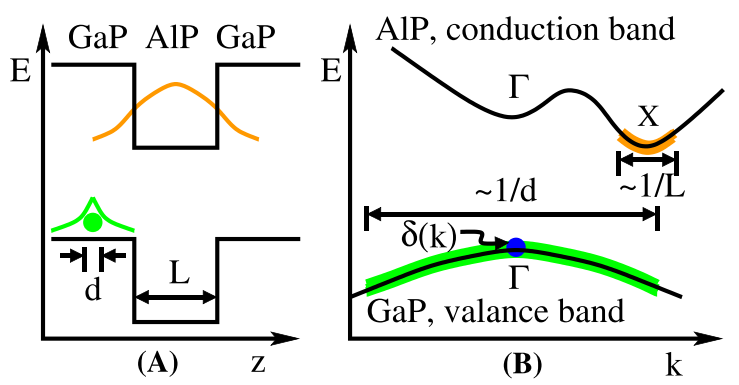

FIG. 7. Schematic representation of confined electron states in AlP QW and localized hole states in GaP in (A) real and (B) momentum space. Spreads in momentum for electron and hole states are related to the inverse of the corresponding localization lengths. Extended hole states would correspond to a momentum $\delta$-function. independently conclude that the A-B(NP) peak at $\sim 2.045 \mathrm{eV}$ is due to localized excitons.

No blue-shift was observed for the A-B peaks in Fig. 4, while B-B peak suffered a characteristic [power] ${ }^{1 / 3}$ blue-shift of type-II transition with increasing excitation power. Absence of blue-shift of the A-B peak with excitation power implies that corresponding states are too strongly localized to be affected by the carrier induced band bending and resultant triangular potential which quantizes the states participating in B-B transitions. The temperature dependence of PL (Fig. 5) where the strength of the $\mathrm{B}-\mathrm{B}(\mathrm{NP})$ peak is enhanced with respect to the bound exciton (A-B) peaks at higher temperature also indicates the "ionization" of the acceptor-bound localized hole states.

Finally, the observation of persistent photoconductivity (in the QW plane) is another independent indication of hole localization. Optical excitation with $532 \mathrm{~nm}$ laser nonresonantly creates electron-hole pairs mainly in the GaP layers. Though some of the photogenerated electrons can be depleted due radiative and nonradiative recombination with holes in GaP layers, a fraction of them would diffuse to the AIP QW layer where the electrons see a lower potential. These QW electrons will control the in-plane photoconductivity of the sample. Depletion of these electrons due to recombination with holes will lead to the decay of photoconductivity. Being a type-II QW system, electron-hole wave function overlap is not optimal for AlP/GaP QWs. Only the holes available near the QW interface can recombine with QW electrons and this cause the initial rapid decay of the photoconductivity in Fig. 6. With the electrons trapped in the QW, a biased diffusion of holes from bulk GaP to the regions close to the $\mathrm{QW}$ interface is expected due to the electrostatic attraction between electrons and holes. If the holes are localized, this transport of holes from the bulk GaP to the heterointerface will be an activated process that is exponentially suppressed at low temperature. This will make the QW electrons available during a very long period of time after their photocreation. Thus, localized holes may lead to persistent photoconductivity. Further study of the dependence of persistent photoconductivity on the sample temperature and excitation laser wavelength may be useful for a more conclusive evidence.

\section{B. Relaxation of momentum selection rule}

Let us now attempt a back-of-the-envelope estimate of the extent of the relaxation of the momentum selection rule due to the localization of holes. The electrons in AlP are at the three equivalent (ignoring the lifted degeneracy) $\mathrm{X}$-valleys, where the wave vectors $\left(k_{x}, k_{y}, k_{z}\right)$ are the three permutations of (001). Furthermore, since the electron confinement in the QW is along the $z$ direction, if we consider only the (001) $\mathrm{X}$-valley electrons of AlP then we have direct transitions for $k_{x}$ and $k_{y}$, and we only need to worry about a mechanism for the relaxation of the momentum conservation rule along the $z$ direction. The problem may thus be effectively treated in one dimension, with only the wave functions along the growth direction $z$ being the important consideration.

The X-valley electrons are QW bound states with a finite momentum $\hbar k_{0}$ along one of the $\vec{k}$ directions depending on 
which of the three X-valleys they belong. For analytic simplicity, let us assume harmonic confinement potentials for electrons and holes resulting in Gaussian wave functions for the ground state electrons and holes [Fig. 8 (inset)]. The ground state wave function for $\mathrm{X}$-valley electrons confined with a confinement length $L$ within the AlP QW thus has the form

$$
\psi_{\mathrm{AlP}}^{X}(z)=(L \pi \sqrt{2 \pi})^{-\frac{1}{2}} \exp \left[-\left(z-z_{0}\right)^{2} / L^{2}-i k_{0} z\right] .
$$

The $\exp \left(-i k_{0} z\right)$ factor, with $k_{0}=\pi / 0.545 \mathrm{~nm}^{-1}$, takes care of the fact that the electrons in AlP are not at the Brillouin zone center and $z_{0}(\neq 0)$ accounts for the type-II nature of the heterostructure since the center of the electron wave function is displaced with respect to the center of the hole wave function. The latter is assumed to be at the origin. The envelope wave function of the acceptor-bound holes with a localization length $d$ in the $z$ direction is taken as

$$
\psi_{\mathrm{GaP}}^{\Gamma}(z)=(d \pi \sqrt{2 \pi})^{-\frac{1}{2}} \exp \left[-z^{2} / d^{2}\right] .
$$

The transition probability $(P)$ is proportional to the square of the overlap integral and can be written as

$$
P \sim\left|\int_{-\infty}^{\infty} \psi_{\mathrm{AlP}}^{X}{ }^{*}(z) \psi_{\mathrm{GaP}}^{\Gamma}(z) d z\right|^{2}
$$

or

$$
\begin{aligned}
P= & \left(\frac{A}{2 \pi\left(\frac{L}{d}+\frac{d}{L}\right)}\right) \times\left(\exp \left[-\frac{2 z_{0}^{2}}{L^{2}+d^{2}}\right]\right) \\
& \times\left(\exp \left[-\frac{k_{0}^{2}}{2\left(\frac{1}{L^{2}}+\frac{1}{d^{2}}\right)}\right]\right) .
\end{aligned}
$$

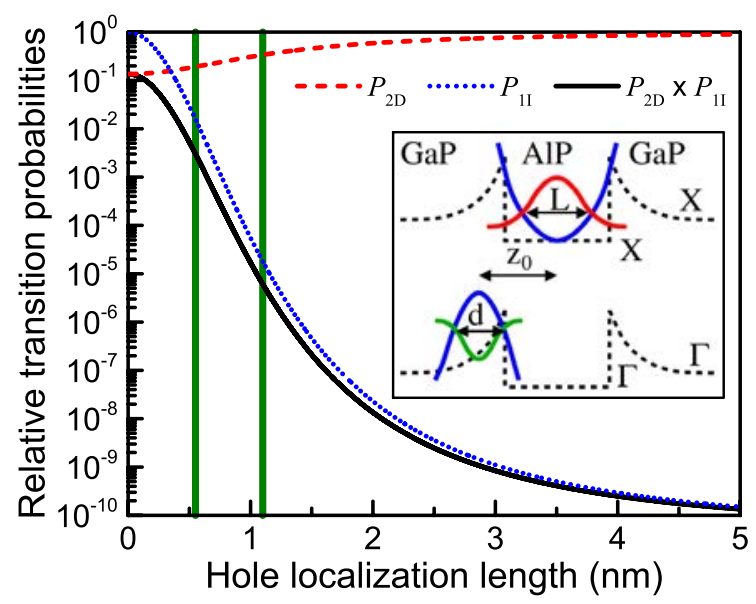

FIG. 8. Numerically calculated transition probabilities for type-II direct $\left(P_{2 \mathrm{D}}\right.$, dashed line $)$, type-I indirect $\left(P_{1 \mathrm{I}}\right.$, dotted line $)$, and type-II indirect $\left(P_{2 \mathrm{D}} \times P_{1 \mathrm{I}}\right.$, solid line $)$ transitions relative to that of type-I direct transition as a function of hole localization length. Vertical solid lines mark the localization length of one and two lattice constants. Inset: Schematic of electron and hole confinement potentials (assumed to be harmonic potentials) and the ground state wave functions (Gaussians).
Here, $A$ is the constant of proportionality. The transition probability neatly factorizes into three terms and may thus be expressed as

$$
P=P_{1 \mathrm{D}} \times P_{2 \mathrm{D}} \times P_{1 \mathrm{I}}
$$

Here, $P_{1 \mathrm{D}}, P_{2 \mathrm{D}}$, and $P_{1 \mathrm{I}}$ correspond to the terms in the three parenthesis, respectively, in Eq. (4). We will only have the first term $P_{1 \mathrm{D}}$ for a type-I direct gap QW. The additional terms $P_{2 \mathrm{D}}$ and $P_{1 \mathrm{I}}$ signify the reduction of the overlap on account of the type-II nature and the off-zone center position of the electrons in the indirect gap AlP.

To make numerical estimate of these effects, we have taken $z_{0}=L$ and $L$ is estimated at $1.2 \mathrm{~nm}$ from the confinement energy ( $\sim 33 \mathrm{meV}$ ) of the ground state electron in AlP QW. Numerical evaluation of various terms in Eq. (5) is plotted in Fig. 8 as a function of hole localization length. Dramatic enhancement in transition probabilities for indirect gap (both type-II and type-I) transitions relative to that of type-I direct gap transition is seen with decreasing hole localization length. As expected, the relative transition probability for type-II direct gap QW increases with increasing hole localization length. The localization length for the acceptor-bound hole is estimated to be one-two lattice constant $(0.55-1.1 \mathrm{~nm})$ in $\mathrm{GaP}$. These positions are marked with thick vertical lines in Fig. 8. In this case, an enhancement of about $10^{5}-10^{8}$ is achieved as compared with the case for unbound holes. Compared with the direct gap type-I QW, the transition probability for a type-II indirect gap transition is still smaller by a factor of $10^{2}-10^{5}$. Note that this calculation is rather approximate. The assumption of Gaussian wave functions leads to an overlap integral having exponential dependence on various parameters, making the results in Fig. 8 extremely sensitive to the parameter values. While having exponential wave functions may be more realistic, the corresponding confinement potentials are no longer simple to model. Harmonic confinement was chosen for analytical simplicity. Experimentally, the intensity of the PL signal from AlP/GaP was about two orders of magnitude smaller than that measured from high quality GaAs/AlGaAs QW.

\section{SUMMARY}

We have shown that there can be relatively efficient light emission from type-II indirect gap GaP/AlP/GaP MQW structures. The observed efficiency of luminescence has been attributed to the breakdown of the momentum selection rule due to the localization of holes by acceptor states close to the GaP/AlP interface. The hole localization energy is estimated at $35 \mathrm{meV}$. Localization of holes is independently established through (i) absence of shift of the low energy no-phonon peak with excitation power, (ii) large intensity of the phonon replica, (iii) temperature dependence of the PL spectrum, and (iv) observation of persistent photoconductivity at low temperature. Furthermore, a true type-II emission, as evidenced by the characteristic [power] ${ }^{1 / 3}$ dependence of the PL peak energy was also established at higher excitation power. 


\section{ACKNOWLEDGMENTS}

B.B. thanks Gottfried Döhler for a useful discussion regarding the selection rules in indirect gap semiconductors. B.P. thanks DST and INSA, the Government of India for partial financial support.

${ }^{1}$ M. Kumagai, T. Takagahara, and E. Hanamura, Phys. Rev. B 37, 898 (1988).

${ }^{2}$ H. Asahi, K. Asami, T. Watanabe, S. J. Yu, T. Kaneko, S. Emura, and S. Gonda, Appl. Phys. Lett. 58, 1407 (1991).

${ }^{3}$ A. Sasaki, X.-L. Wang, and A. Wakahara, Appl. Phys. Lett. 64, 2016 (1994).

${ }^{4}$ K. Uchida, N. Miura, J. Kitamura, and H. Kukimoto, Phys. Rev. B 53, 4809 (1996).

${ }^{5}$ R. K. Soni, S. Tripathy, and H. Asahi, Physica E 21, 131 (2004).

${ }^{6}$ C. H. Park and K. J. Chang, Phys. Rev. B 47, 12709 (1993).

${ }^{7}$ S. Nagao, T. Fujimori, H. Gotoh, H. Fukushima, T. Takano, H. Ito, S. Koshihara, and F. Minami, J. Appl. Phys. 81, 1417 (1997).

${ }^{8}$ S. Nagao, K. Fujii, T. Fujimori, H. Gotoh, H. Ito, and F. Minami, J. Cryst. Growth 175/176, 1157 (1997).

${ }^{9}$ F. Issiki, S. Fukatsu, and Y. Shiraki, Appl. Phys. Lett. 67, 1048 (1995).

${ }^{10}$ N. Usami, F. Issiki, D. K. Nayak, Y. Shiraki, and S. Fukatsu, Appl. Phys. Lett. 67, 524 (1995).
${ }^{11}$ N. Usami, T. Sugita, T. Ohta, F. Issiki, Y. Shiraki, K. Uchida, and N. Miura, Phys Rev B 60, 1879 (1999).

${ }^{12}$ K. Arimoto, T. Sugita, N. Usami, and Y. Shiraki, Phys. Rev. B 60, 13735 (1999).

${ }^{13}$ M. Gerhold, K. Kamath, and P. Bhattacharya, Appl. Phys. Lett. 71, 3260 (1997).

${ }^{14}$ M. P. Semtsiv, U. Müller, W. T. Masselink, N. Georgiev, T. Dekorsy, and M. Helm, Appl. Phys. Lett 89, 184102 (2006).

${ }^{15}$ M. P. Semtsiv, O. Bierwagen, W. T. Masselink, M. Goiran, J. Galibert, and J. Léotin, Phys. Rev. B 77, 165327 (2008).

${ }^{16}$ M. P. Semtsiv, S. Dressler, W. T. Masselink, V. V. Rylkov, J. Galibert, M. Goiran, and J. Léotin, Phys. Rev. B 74, 041303(R) (2006).

${ }^{17}$ N. N. Ledentsov, J. Böhrer, M. Beer, F. Heinrichsdorff, M. Grundmann, D. Bimberg, S. V. Ivanov, B. Ya. Meltser, S. V. Shaposhnikov, I. N. Yassievich, N. N. Faleev, P. S. Kop'ev, and Zh. I. Alferov, Phys. Rev. B 52, 14058 (1995).

${ }^{18}$ B. Pödör, Phys. Status Solidi B 120, 207 (1983).

${ }^{19}$ B. Pal, K. Goto, M. Ikezawa, Y. Masumoto, P. Mohan, J. Motohisa, and T. Fukui, Appl. Phys. Lett. 93, 073105 (2008).

${ }^{20}$ T. T. Chen, W. S. Su, Y. F. Chen, P. W. Liu, and H. H. Lin, Appl. Phys. Lett. 85, 1526 (2004).

${ }^{21}$ Energy position of the B-B(NP) energy is estimated from the spectra at the lowest excitation power where it becomes decipherable.

${ }^{22}$ B. Bansal, A. Kadir, A. Bhattacharya, and V. V. Moshchalkov, Appl. Phys. Lett. 93, 021113 (2008).

${ }^{23}$ I. Brener, M. Olszakier, E. Cohen, E. Ehrenfreund, A. Ron, and L. Pfeiffer, Phys. Rev. B 46, 7927 (1992). 